\title{
Drug Utilization and Medical Cost Study Focusing on Moisturizers in Cancer Patients Treated With Molecular Targeted Therapy: a Retrospective Observational Study Using Data From a Japanese Claims Database
}

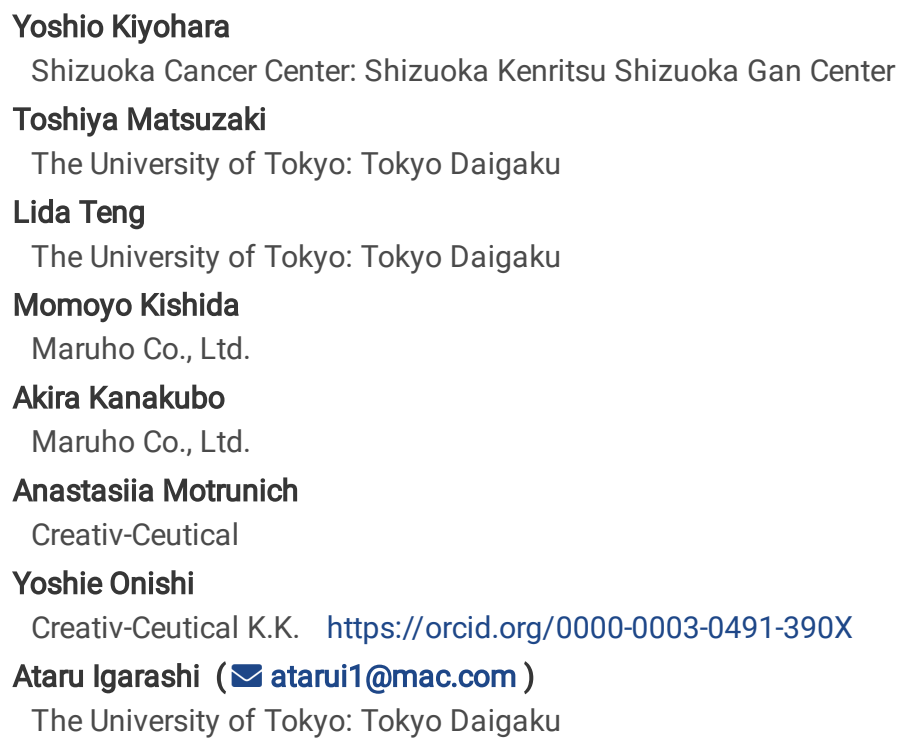

\section{Research Article}

Keywords: Molecular targeted therapy, Dry skin, Heparinoid, Moisturizer, Medical cost, Claims data analysis

Posted Date: October 20th, 2021

DOI: https://doi.org/10.21203/rs.3.rs-882012/v1

License: (c) (i) This work is licensed under a Creative Commons Attribution 4.0 International License. Read Full License

Version of Record: A version of this preprint was published at Dermatology and Therapy on April 10th, 2022. See the published version at https://doi.org/10.1007/s13555-022-00712-2. 


\section{Abstract}

Purpose Molecular targeted therapies (MTTs) cause skin disorders in cancer patients, and moisturizers are useful treatments; however, their actual use and costs are unknown. Our purpose was to examine the use and costs of moisturizers prescribed for xerosis (asteatosis) in cancer patients treated with MTTs.

Methods We used data from a Japanese hospital-based claims database. The index date was the first date of MTT prescription from October 2011 to April 2018 (selection period), and the follow-up period was 1 year from the index date. Patients treated with MTTs during the selection period and who were not prescribed moisturizers in the 6 months before the index date were included as the study cohort. Timing, duration, amount, and costs of the prescribed moisturizers and total medical costs were analyzed.

Results Among the 78,190 patients in the study cohort, 27,906 patients (35.7\%) were prescribed moisturizers during follow-up. Moisturizer prescription timing, duration, and volume were inconsistent. The average annual total medical costs for treating patients with MTT who were prescribed moisturizers was 6.165 million yen per patient, and the moisturizer costs were 6033 yen. The number of patients who used moisturizers showed an increasing trend.

Conclusion No consistent patterns were observed for the timing or duration of moisturizer use, which suggests various developmental patterns of skin disorders. Furthermore, medical costs for moisturizers accounted for only a small proportion of the total medical costs required for cancer treatment.

\section{Introduction}

Among anti-cancer drugs, molecular targeted therapies (MTTs), particularly epidermal growth factor receptor (EGFR) inhibitors, multikinase inhibitors, and BCR-ABL tyrosine kinase inhibitors, may cause skin disorders as an adverse reaction. Patients may develop hand-foot syndrome (HFS), xerosis (asteatosis), and acneiform rash, and moisturizers are used as treatments [1]. Clinical practice guidance in Japan [2] evaluates the topical application of moisturizers for HFS, xerosis, and acneiform rash caused by treatment with MTTs. Topical application of moisturizers is a grade B recommendation (recommendable with evidence) for HFS and is considered "useful and recommended." The recommendation grade for xerosis is $\mathrm{C1a}$ (can be used, but, because of insufficient scientific evidence, is less recommendable than therapies with evidence), and "the use of moisturizers taking advantage of their characteristics is recommended in order to improve dermatitis and subjective symptoms." The recommendation grade for acneiform rash is $\mathrm{C} 1 \mathrm{~b}$ (no evidence, but the use is not denied), and the guidance states that "the topical application of moisturizers may be considered." Case series describing the clinical usefulness of topical agents in patients being treated with MTTs have been reported [3, 4]; however, there are no controlled studies.

The usefulness of heparinoids, petrolatum, and urea, which are used as prescription moisturizers in Japan, has been reported for the treatment of both primary xerosis and xerosis caused by other diseases, drugs, and therapies; for example, dryness owing to MTT [4], radiation dermatitis associated with radiotherapy [5,6], and dryness and pruritus in patients on dialysis [7]. Prescribed moisturizers contribute to adherence to topical therapy because physicians, nurses, and pharmacists explain directly to the patients the necessity of moisturizers and how to apply them. Treatment of xerosis caused by cancer treatment tends to be neglected relative to the treatment of the underlying disease. However, the therapeutic effect of EGFR inhibitors is higher in patients who develop skin disorders [8, 9]. Therefore, the treatment of skin disorders conceivably contributes to the continuation of treatment with MTT. However, the current status of moisturizer use is unclear.

The purpose of this study was to analyze the utilization patterns and medical costs of moisturizers in patients treated with MTT, using a health insurance claims database.

\section{Methods}

This was a retrospective observational study.

\section{Data source}

We used the Japanese hospital-based claims database developed by Medical Data Vision, Co., Ltd. (MDV; Tokyo, Japan) for our analyses. The MDV database comprises approximately 28 million patients from 400 hospitals in Japan that use the Diagnosis Related Group-like fixed payment system, which is called the Diagnosis Procedure Combination (DPC) system in Japan. These hospitals account for $22 \%$ of all acutephase hospitals and are widely distributed throughout Japan. The MDV database uses an anonymized patient identifier and stores the following patient information: sex, birth year, date of medical service, diagnosis codes, hospitalization, medical procedures, test orders, operations, and prescriptions.

The source cohort in this study comprised patients who were prescribed any MTT (Online Resource 1) between April 2008 and April 2019 ( $n=$ $190,536)$ with continuous enrollment for at least 12 months. 
The patient selection period was from October 2011 to April 2018. The follow-up period was 1 year from the index date, which was defined as the first prescription date of MTT, and the pre-index period was defined as the 6-month period before the index date. We presumed that it would take 56 days from the end of anti-cancer drug use for the dermatological effects of the drug to resolve, and we considered this period the washout period. The study cohort was determined by evaluating patients according to the inclusion and exclusion criteria.

Inclusion criteria:

Patients who were treated with the MTTs listed in Online Resource 1 during the patient selection period.

Exclusion criteria:

Patients to whom any of the following conditions applied:

- not treated with the defined MTTs during the patient selection period;

- prescribed at least one moisturizer (heparinoids, petrolatum, urea) within the pre-index period;

- prescribed at least one MTT within the pre-index period;

- not continuously observed in the database during the follow-up period (patients must have had at least one medical claim each quarter within the follow-up period);

- age < 18 years at the index date; and

- prescribed MTT drugs from more than one MTT category by the index date.

\section{Patient characteristics}

The following patient characteristics data were collected:

- age at the index date;

- sex;

- cancer type (International Classification of Diseases version 10 (ICD-10) codes: C00-C96);

- type of MTT being used; and

- type of moisturizer (Anatomical, Therapeutic, Chemical Classification (ATC) codes: C05B0 [heparinoid], D02A0 [urea], and V07A0 [petrolatum]).

\section{Statistical analysis}

The following variables describing the use of moisturizers were analyzed using descriptive statistics:

- number of days for which a patient was treated with MTT during the follow-up period;

- total number of days for which a patient used moisturizers during the follow-up period, total amount (g) of prescribed moisturizers, and the number and proportion of patients who were prescribed moisturizers (by month);

- total number of days for which a patient used moisturizers after the washout period, total amount ( $\mathrm{g}$ ) of prescribed moisturizers, and the number and proportion of patients who were prescribed moisturizers during the follow-up period;

- whether treatment with radiotherapy was performed during the follow-up period (according to the presence of the management/implementation fee for radiotherapy, M001), and if so, the number of days a patient received treatment; and

- total medical costs 6 months and 12 months from the index date, and costs of outpatient visits, hospitalization, prescribed drugs, and radiotherapy.

Descriptive analyses were performed using standard descriptive statistics. For continuous variables, sample sizes ( $n$ ), measures of central tendency (mean, median), and measures of variation (standard deviation, minimum, maximum, quartiles, and/or percentiles) were provided. For categorical variables, frequency tables containing sample sizes and proportions (\%) for the different categories were presented. We also performed analyses stratified by heparinoids, petrolatum, and urea. All analyses were performed using SAS software version 9.3 (SAS Institute, Cary, NC, USA). Note that the number of prescription days for moisturizers was set at 28 days for each moisturizer prescription. If there were multiple prescriptions, and the interval between prescription dates was less than 28 days, the number of prescription days for the moisturizer was set to the number of days between prescription dates.

\section{Results}


Of 190,536 patients in the source cohort, 78,190 patients were included in the study cohort. Among the study cohort, 27,906 patients (35.7\%) were prescribed moisturizers (Group M), and 50,284 patients (64.3\%) were not prescribed moisturizers (Group N) (Fig. 1).

\section{Patient demographics}

Table 1 shows the patients' background characteristics, groups according to the use of moisturizers, and groups divided by the type of moisturizer (heparinoids, petrolatum, urea, and a combination of two or more moisturizers). When Group N (50,284 patients) was compared with Group M (27,906 patients), the proportion of men was slightly higher vs women ( $47.4 \%$ vs. $45.4 \%$, respectively), and the mean age was also slightly higher (65.7 years vs. 64.7 years, respectively) in Group M. Also in Group M, users of heparinoids accounted for $72.6 \%$ ( $n=20,265)$ of the patients; petrolatum: $14.2 \%(n=3960)$; urea: $10.6 \%(n=2952)$, and a combination of moisturizers: $2.6 \%(n=729)$. Among the MTT groups with $\geq$ 1000 patients, the proportion of moisturizer users was highest in the EGFR inhibitor group (71.4\%), followed by the multikinase inhibitor group (50.2\%), and the immune check point inhibitor group (42.1\%), and lowest in the Janus kinase (JAK) inhibitor group (10.7\%), BCR-ABL inhibitor group (15.4\%), and anti-cluster of differentiation 20 (CD20) antibody group (23.2\%) (Table 2).

Table 1

Baseline characteristics (at the index date) for the patient cohort

\begin{tabular}{|c|c|c|c|c|c|c|c|c|c|c|c|c|c|c|}
\hline & \multicolumn{2}{|l|}{ Overall } & \multicolumn{2}{|c|}{$\begin{array}{l}\text { All } \\
\text { (no moisturizer) }\end{array}$} & \multicolumn{2}{|c|}{$\begin{array}{l}\text { All } \\
\text { (with } \\
\text { moisturizer) }\end{array}$} & \multicolumn{2}{|c|}{ Heparinoid } & \multicolumn{2}{|c|}{ Petrolatum } & \multicolumn{2}{|l|}{ Urea } & \multicolumn{2}{|c|}{ Combination } \\
\hline & \multicolumn{2}{|l|}{78,190} & 50,284 & $64.3 \%$ & 27,906 & $35.7 \%$ & 20,265 & $72.6 \%$ & 3,960 & $14.2 \%$ & 2,952 & $10.6 \%$ & 729 & $2.6 \%$ \\
\hline \multicolumn{15}{|l|}{$\begin{array}{l}\text { Sex, n } \\
(\%)\end{array}$} \\
\hline Male & 36,041 & $46.1 \%$ & 22,805 & $45.4 \%$ & 13,236 & $47.4 \%$ & 9588 & $47.3 \%$ & 1841 & $46.5 \%$ & 1481 & $50.2 \%$ & 326 & $44.7 \%$ \\
\hline Female & 42,149 & $53.9 \%$ & 27,479 & $54.7 \%$ & 14,670 & $52.6 \%$ & 10,677 & $52.7 \%$ & 2119 & $53.5 \%$ & 1471 & $49.8 \%$ & 403 & $55.3 \%$ \\
\hline \multicolumn{15}{|l|}{ Age } \\
\hline $\begin{array}{l}\text { Mean } \\
\text { (SD) }\end{array}$ & \multicolumn{2}{|c|}{65.07 (12.25) } & \multicolumn{2}{|c|}{$64.71(12.50)$} & \multicolumn{2}{|c|}{65.73 (11.76) } & \multicolumn{2}{|c|}{65.57 (11.67) } & \multicolumn{2}{|c|}{66.15 (12.34) } & \multicolumn{2}{|c|}{66.41 (11.47) } & \multicolumn{2}{|c|}{$\begin{array}{l}65.13 \\
(12.05)\end{array}$} \\
\hline $95 \% \mathrm{Cl}$ & \multicolumn{2}{|c|}{$64.99-65.16$} & \multicolumn{2}{|c|}{$64.60-64.82$} & \multicolumn{2}{|c|}{$65.59-65.87$} & \multicolumn{2}{|c|}{$65.41-65.73$} & \multicolumn{2}{|c|}{$65.77-66.54$} & \multicolumn{2}{|c|}{$65.99-66.82$} & \multicolumn{2}{|c|}{$64.26-66.01$} \\
\hline $\begin{array}{l}\text { Min, } \\
\text { Max }\end{array}$ & \multicolumn{2}{|l|}{18,99} & \multicolumn{2}{|l|}{18,98} & \multicolumn{2}{|l|}{18,99} & \multicolumn{2}{|l|}{18,97} & \multicolumn{2}{|l|}{18,99} & \multicolumn{2}{|l|}{18,94} & \multicolumn{2}{|c|}{18,90} \\
\hline Median & \multicolumn{2}{|l|}{67} & \multicolumn{2}{|l|}{66} & 67 & & 67 & & 68 & & 68 & & 66 & \\
\hline $\begin{array}{l}\text { Age } \\
\text { category } \\
\text { (years), } \\
\text { n (\%) }\end{array}$ & & & & & & & & & & & & & & \\
\hline $18-34$ & 1358 & $1.7 \%$ & 980 & $2.0 \%$ & 378 & $1.4 \%$ & 255 & $1.3 \%$ & 78 & $2.0 \%$ & 30 & $1.0 \%$ & 15 & $2.1 \%$ \\
\hline $35-44$ & 3970 & $5.1 \%$ & 2726 & $5.4 \%$ & 1244 & $4.5 \%$ & 923 & $4.6 \%$ & 168 & $4.2 \%$ & 115 & $3.9 \%$ & 38 & $5.2 \%$ \\
\hline $45-54$ & 9099 & $11.6 \%$ & 6102 & $12.1 \%$ & 2997 & $10.7 \%$ & 2212 & $10.9 \%$ & 416 & $10.5 \%$ & 302 & $10.2 \%$ & 67 & $9.2 \%$ \\
\hline $55-65$ & 18,552 & $23.7 \%$ & 12,023 & $23.9 \%$ & 6529 & $23.4 \%$ & 4778 & $23.6 \%$ & 866 & $21.9 \%$ & 691 & $23.4 \%$ & 194 & $26.6 \%$ \\
\hline $65-75$ & 27,301 & $34.9 \%$ & 17,144 & $34.1 \%$ & 10,157 & $36.4 \%$ & 7483 & $36.9 \%$ & 1375 & $34.7 \%$ & 1049 & $35.5 \%$ & 250 & $34.3 \%$ \\
\hline $75+$ & 17,910 & $22.9 \%$ & 11,309 & $22.5 \%$ & 6601 & $23.7 \%$ & 4614 & $22.8 \%$ & 1057 & $26.7 \%$ & 765 & $25.9 \%$ & 165 & $22.6 \%$ \\
\hline
\end{tabular}


Table 2

Number of patients and the proportion of moisturizer users by MTT group

\begin{tabular}{|c|c|c|c|c|c|c|c|c|c|c|c|c|c|}
\hline & \multirow[t]{2}{*}{ Overall } & \multirow{2}{*}{\multicolumn{2}{|c|}{$\begin{array}{l}\text { All } \\
\text { (no moisturizer) }\end{array}$}} & \multirow{2}{*}{\multicolumn{2}{|c|}{$\begin{array}{l}\text { All } \\
\text { (with } \\
\text { moisturizer) }\end{array}$}} & \multicolumn{8}{|c|}{ details of moisturizer } \\
\hline & & & & & & \multicolumn{2}{|c|}{ Heparinoid } & \multicolumn{2}{|c|}{ Petrolatum } & \multicolumn{2}{|l|}{ Urea } & \multicolumn{2}{|c|}{ Combination } \\
\hline \multicolumn{14}{|c|}{ MTT group (at the index date), $n$ (\% of usage in the MTT group) } \\
\hline VEGF inhibitor & 19,728 & 11,804 & $59.8 \%$ & 7924 & $40.2 \%$ & 6060 & $30.7 \%$ & 869 & $4.4 \%$ & 782 & $4.0 \%$ & 213 & $1.1 \%$ \\
\hline $\begin{array}{l}\text { Anti CD20 } \\
\text { antibody }\end{array}$ & 17,568 & 13,492 & $76.8 \%$ & 4076 & $23.2 \%$ & 2465 & $14.0 \%$ & 1087 & $6.2 \%$ & 449 & $2.6 \%$ & 75 & $0.4 \%$ \\
\hline HER2 inhibitor & 12,026 & 8099 & $67.3 \%$ & 3927 & $32.7 \%$ & 3000 & $24.9 \%$ & 440 & $3.7 \%$ & 389 & $3.2 \%$ & 98 & $0.8 \%$ \\
\hline EGFR inhibitor & 7177 & 2056 & $28.6 \%$ & 5121 & $71.4 \%$ & 4180 & $58.2 \%$ & 373 & $5.2 \%$ & 391 & $5.4 \%$ & 177 & $2.5 \%$ \\
\hline BCR-ABL inhibitor & 6387 & 5406 & $84.6 \%$ & 981 & $15.4 \%$ & 668 & $10.5 \%$ & 185 & $2.9 \%$ & 112 & $1.8 \%$ & 16 & $0.3 \%$ \\
\hline $\begin{array}{l}\text { Proteasome } \\
\text { inhibitors }\end{array}$ & 4031 & 2715 & $67.4 \%$ & 1316 & $32.6 \%$ & 757 & $18.8 \%$ & 358 & $8.9 \%$ & 177 & $4.4 \%$ & 24 & $0.6 \%$ \\
\hline $\begin{array}{l}\text { Immune check } \\
\text { point inhibitor }\end{array}$ & 2699 & 1564 & $57.9 \%$ & 1135 & $42.1 \%$ & 918 & $34.0 \%$ & 153 & $5.7 \%$ & 47 & $1.7 \%$ & 17 & $0.6 \%$ \\
\hline $\begin{array}{l}\text { Multikinase } \\
\text { inhibitor }\end{array}$ & 2501 & 1246 & $49.8 \%$ & 1255 & $50.2 \%$ & 744 & $29.7 \%$ & 106 & $4.2 \%$ & 342 & $13.7 \%$ & 63 & $2.5 \%$ \\
\hline VEGFR inhibitor & 2067 & 1242 & $60.1 \%$ & 825 & $39.9 \%$ & 603 & $29.2 \%$ & 91 & $4.4 \%$ & 113 & $5.5 \%$ & 18 & $0.9 \%$ \\
\hline mTOR inhibitor & 1186 & 732 & $61.7 \%$ & 454 & $38.3 \%$ & 313 & $26.4 \%$ & 54 & $4.6 \%$ & 79 & $6.7 \%$ & 8 & $0.7 \%$ \\
\hline ALK inhibitor & 774 & 614 & $79.3 \%$ & 160 & $20.7 \%$ & 129 & $16.7 \%$ & 18 & $2.3 \%$ & 9 & $1.2 \%$ & 4 & $0.5 \%$ \\
\hline Retinoid & 561 & 300 & $53.5 \%$ & 261 & $46.5 \%$ & 114 & $20.3 \%$ & 116 & $20.7 \%$ & 26 & $4.6 \%$ & 5 & $0.9 \%$ \\
\hline JAK inhibitor & 457 & 408 & $89.3 \%$ & 49 & $10.7 \%$ & 35 & $7.7 \%$ & 13 & $2.8 \%$ & 1 & $0.2 \%$ & 0 & $0.0 \%$ \\
\hline $\begin{array}{l}\text { Antibody-drug } \\
\text { conjugate (ADC) }\end{array}$ & 380 & 244 & $64.2 \%$ & 136 & $35.8 \%$ & 94 & $24.7 \%$ & 26 & $6.8 \%$ & 9 & $2.4 \%$ & 7 & $1.8 \%$ \\
\hline CDK 4/6 inhibitor & 299 & 203 & $67.9 \%$ & 96 & $32.1 \%$ & 66 & $22.1 \%$ & 21 & $7.0 \%$ & 7 & $2.3 \%$ & 2 & $0.7 \%$ \\
\hline $\begin{array}{l}\text { Anti-CCR4 } \\
\text { antibody }\end{array}$ & 215 & 54 & $25.1 \%$ & 161 & $74.9 \%$ & 98 & $45.6 \%$ & 44 & $20.5 \%$ & 18 & $8.4 \%$ & 1 & $0.5 \%$ \\
\hline $\begin{array}{l}\text { Anti-SLAMF7 } \\
\text { antibody }\end{array}$ & 55 & 44 & $80.0 \%$ & 11 & $20.0 \%$ & 9 & $16.4 \%$ & 2 & $3.6 \%$ & 0 & $0.0 \%$ & 0 & $0.0 \%$ \\
\hline BTK inhibitor & 49 & 40 & $81.6 \%$ & 9 & $18.4 \%$ & 7 & $14.3 \%$ & 0 & $0.0 \%$ & 1 & $2.0 \%$ & 1 & $2.0 \%$ \\
\hline $\begin{array}{l}\text { Anti-CD38 } \\
\text { antibody }\end{array}$ & 15 & 13 & $86.7 \%$ & 2 & $13.3 \%$ & 2 & $13.3 \%$ & 0 & $0.0 \%$ & 0 & $0.0 \%$ & 0 & $0.0 \%$ \\
\hline $\begin{array}{l}\text { Histone } \\
\text { deacetylase } \\
\text { inhibitors }\end{array}$ & 11 & 5 & $45.5 \%$ & 6 & $54.5 \%$ & 3 & $27.3 \%$ & 3 & $27.3 \%$ & 0 & $0.0 \%$ & 0 & $0.0 \%$ \\
\hline $\begin{array}{l}\text { Anti-CD52 } \\
\text { antibody }\end{array}$ & 3 & 2 & $66.7 \%$ & 1 & $33.3 \%$ & 0 & $0.0 \%$ & 1 & $33.3 \%$ & 0 & $0.0 \%$ & 0 & $0.0 \%$ \\
\hline BRAF inhibitor & 1 & 1 & $100.0 \%$ & 0 & $0.0 \%$ & 0 & $0.0 \%$ & 0 & $0.0 \%$ & 0 & $0.0 \%$ & 0 & $0.0 \%$ \\
\hline $\begin{array}{l}\text { Bispecific CD19- } \\
\text { directed CD3 T cell } \\
\text { engager antibody } \\
\text { construct }\end{array}$ & 0 & 0 & - & 0 & - & 0 & - & 0 & - & 0 & - & 0 & - \\
\hline FLT3-AXL inhibitor & 0 & 0 & - & 0 & - & 0 & - & 0 & - & 0 & - & 0 & - \\
\hline
\end{tabular}




\begin{tabular}{|c|c|c|c|c|c|c|c|c|c|c|c|c|c|}
\hline \multirow[b]{3}{*}{ MEK inhibitor } & \multirow{3}{*}{$\begin{array}{l}\text { Overall } \\
0\end{array}$} & \multirow{2}{*}{\multicolumn{2}{|c|}{$\begin{array}{l}\text { All } \\
\text { (no moisturizer) }\end{array}$}} & \multirow{2}{*}{\multicolumn{2}{|c|}{$\begin{array}{l}\text { All } \\
\text { (with } \\
\text { moisturizer) }\end{array}$}} & \multicolumn{8}{|c|}{ details of moisturizer } \\
\hline & & & & & & \multicolumn{2}{|c|}{ Heparinoid } & \multicolumn{2}{|c|}{ Petrolatum } & \multicolumn{2}{|c|}{ Urea } & \multicolumn{2}{|c|}{ Combination } \\
\hline & & 0 & - & 0 & - & 0 & - & 0 & - & 0 & - & 0 & - \\
\hline PARP inhibitor & 0 & 0 & - & 0 & - & 0 & - & 0 & - & 0 & - & 0 & - \\
\hline $\begin{array}{l}\text { Radioisotope- } \\
\text { labeled } \\
\text { antibody }\end{array}$ & 0 & 0 & - & 0 & - & 0 & - & 0 & - & 0 & - & 0 & - \\
\hline $\begin{array}{l}\text { EGFR, epiderma } \\
\text { vascular endotl } \\
\text { lymphocytic ac } \\
\text { anaplastic lym } \\
\text { signalregulated }\end{array}$ & $\begin{array}{l}\text { th factor } \\
\text { rowth fa } \\
\text { molecu } \\
\text { kinase; } \\
; C D K, 4\end{array}$ & & $\begin{array}{l}\text { 2, human } \\
\text { EGF recep } \\
\text {, BCR-AB } \\
\text { rosine ki }\end{array}$ & iiler & $\begin{array}{l}\text { growt } \\
\text { ster o } \\
\text { e; } F L T \text {. } \\
\text { Janus }\end{array}$ & & $\begin{array}{l}\text { eptor; } \\
\text { tion; } \\
\text {-like t } \\
A F, B-\end{array}$ & & $3 / \mathrm{re}$ & & & $e$ & $\begin{array}{l}\text { íling } \\
\text { ALK } \\
\text { lular }\end{array}$ \\
\hline
\end{tabular}

Among 78,190 patients who were treated with MTT, the most common cancer codes were C81-96 "malignant neoplasms, stated or presumed to be primary, of lymphoid, haematopoietic and related tissue" (34.7\%), when codes C76-80 "malignant neoplasms of ill-defined, secondary and unspecified sites" were excluded (Online Resource 2). Moreover, when the types of cancer with $\geq 1000$ patients (10 types) were compared, the proportion of moisturizer users in MTT group was high in patients with respiratory system (47.9\%), digestive system (45.7\%), and urinary system (45.6\%) cancer and low in patients with breast (32.2\%), female genital (27.2\%), and lymphoid tissue (24.1\%) cancer.

Moisturizer use

\section{Number of days patients were treated with anti-cancer drugs (MTTs) during the follow-up period}

Regarding the number of days patients were treated with MTTs during the follow-up period, data were collected according to MTT category and were further divided into an oral drug group and an injectable drug group (Online Resource 3). In the EGFR inhibitor group, one of the groups in which the proportion of moisturizer users was the highest, moisturizer users had more treatment days for both oral drugs ( 252.1 days vs. 225.5 days) and injectable drugs ( 11.9 days vs. 9.0 days) compared with patients who did not use moisturizers. In both Group M and Group N, many anti-cancer drugs were prescribed for a different number of days. However, no consistent pattern was observed in the prescription length.

Total number of days for which a patient used moisturizers during the follow-up period, total amount (g) of prescribed moisturizers, and the number and proportion of patients who were prescribed moisturizers by month

The prescription length in days and the amount of moisturizers prescribed during the follow-up period are shown in Table 3. The mean amount of heparinoids (22,537 patients) administered was $393.6 \mathrm{~g}$ (median, $150 \mathrm{~g}$ ), and the mean prescription length was 92.2 days (median, 56 days). The mean amount of petrolatum (6615 patients) administered was $127.8 \mathrm{~g}$ (median, $50 \mathrm{~g}$ ), and the mean prescription length was 48.5 days (median, 28 days). The mean amount of urea formulations (4356 patients) administered was $174.4 \mathrm{~g}$ (median, $80 \mathrm{~g}$ ), and the mean prescription length was 70.3 days (median, 47 days). 
Table 3

Proportion of patients, quantity, and prescription days of heparinoids/petrolatum/urea in all patients receiving moisturizers

\begin{tabular}{|c|c|c|c|c|c|c|}
\hline & \multirow[t]{3}{*}{$n(\%)$} & & \multicolumn{2}{|l|}{ Quantity (g) } & \multicolumn{2}{|c|}{ Period (days) } \\
\hline & & & Mean (SD) & Median & Mean (SD) & Median \\
\hline & & & & (Q1-Q3) & & (Q1-Q3) \\
\hline Heparinoids & 22,537 & $80.8 \%$ & $393.6(692.8)$ & $150(60-430)$ & $92.2(79.4)$ & $56(28-127)$ \\
\hline Petrolatum & 6615 & $23.7 \%$ & $127.8(304.5)$ & $50(20-100)$ & $48.5(42.3)$ & $28(28-56)$ \\
\hline Urea & 4356 & $15.6 \%$ & $174.4(293.5)$ & $80(40-200)$ & $70.3(64.4)$ & $47(28-84)$ \\
\hline \multicolumn{7}{|c|}{$\begin{array}{l}\% \text { indicates the percentage of the total number of patients receiving moisturizers calculated cumulatively from the index date up to the end of } \\
\text { follow-up }\end{array}$} \\
\hline \multicolumn{7}{|c|}{$n$, number; $S D$, standard deviation; $Q 1-Q 3$, first to third quartile } \\
\hline
\end{tabular}

For patients who were prescribed moisturizers, the timing of the start of the moisturizer prescription after the MTT prescription date and the number of patients who were prescribed moisturizers at each time point are shown in Online Resource 4. Moisturizers were prescribed for 31.5\% of the patients within 1 month from the start of MTT prescriptions, for $74.0 \%$ within 6 months, and $100 \%$ within 12 months. According to the inclusion criteria, patients whose prescriptions were started after the 12th month were not included in the study cohort.

\section{Total number of days for which a patient used moisturizers after the washout period and the total amount (g) of prescribed moisturizers}

The prescribed amount of each moisturizer and the prescription length in days after the washout period were calculated as during the follow-up period. The mean amount of heparinoids (3932 patients) administered was $213.5 \mathrm{~g}$ (median, $100 \mathrm{~g}$ ), and the mean prescription length was 53.1 days (median, 31 days). The mean amount of petrolatum (1335 patients) administered was $135.5 \mathrm{~g}$ (median, $50 \mathrm{~g}$ ), and the mean prescription length was 39.4 days (median, 28 days). The mean amount of urea formulations (657 patients) administered was $121.9 \mathrm{~g}$ (median, $60 \mathrm{~g}$ ), and the mean prescription length was 47.4 days (median, 28 days).

Treatment with radiotherapy during the follow-up period (medical remuneration points and management/implementation fee for radiotherapy, code: M001)

The number of patients treated with radiotherapy during the follow-up period and the number of days for which patients received treatment are shown in Online Resource 5. In the study cohort, $10.0 \%$ of the patients (7801 patients) received concomitant radiotherapy, and the mean length of treatment was 22.8 days (median, 20 days). The proportion of moisturizer users was higher in patients who received radiotherapy than in those who did not receive radiotherapy.

Total medical costs 6 months and 12 months from the index date, and the costs of outpatient visits, hospitalization, drugs (MTTs, other drugs, and moisturizers), and radiotherapy

When the total medical costs of cancer treatment were calculated by item over the 6-month and 12-month periods after the index date, medical costs for Group M were higher than for Group N by 467,000 yen (3.766 million vs. 3.299 million) at 6 months and 1.029 million yen (6.165 million yen vs. 5.135 million yen) at 12 months (Table 4). In Group M, the moisturizer cost was 4008 yen at 6 months and 6033 yen at 12 months, which accounted for approximately $0.1 \%$ of the total medical costs. The moisturizer costs accounted for $0.86 \%$ of the difference between the two groups at 6 months and $0.59 \%$ at 12 months. 
Table 4

Cost (in Japanese yen) analysis during the first 6 months of follow-up and for the entire 12 months of follow-up

\begin{tabular}{|c|c|c|c|c|c|c|c|c|}
\hline & \multicolumn{4}{|l|}{ Group N } & \multicolumn{4}{|c|}{ Group M } \\
\hline & $n$ & Mean (SD) & $95 \% \mathrm{Cl}$ & $\begin{array}{l}\% \text { of total } \\
\text { medical } \\
\text { costs }\end{array}$ & $n$ & Mean (SD) & $95 \% \mathrm{Cl}$ & $\begin{array}{l}\% \text { of total } \\
\text { medical } \\
\text { costs }\end{array}$ \\
\hline \multicolumn{9}{|c|}{ First 6 months of follow-up } \\
\hline $\begin{array}{l}\text { Total medical } \\
\text { costs }\end{array}$ & 50,284 & $\begin{array}{l}3,298,769 \\
(2,069,605)\end{array}$ & $\begin{array}{l}3280679- \\
3316859\end{array}$ & & 27,906 & $\begin{array}{l}3,766,163 \\
(2,317,206)\end{array}$ & $\begin{array}{l}3,738,975- \\
3,793,352\end{array}$ & \\
\hline $\begin{array}{l}\text { Outpatient } \\
\text { costs }\end{array}$ & 49,598 & $\begin{array}{l}2,126,359 \\
(1,729,972)\end{array}$ & $\begin{array}{l}2,111,134- \\
2,141,584\end{array}$ & $64.5 \%$ & 27,246 & $\begin{array}{l}2,087,803 \\
(1,764,503)\end{array}$ & $\begin{array}{l}2,066,851- \\
2,108,756\end{array}$ & $55.4 \%$ \\
\hline Inpatient costs & 32,117 & $\begin{array}{l}1,881,002 \\
(1,948,841)\end{array}$ & $\begin{array}{l}1,859,688- \\
1,902,317\end{array}$ & $57.0 \%$ & 20,912 & $\begin{array}{l}2,305,579 \\
(2,393,697)\end{array}$ & $\begin{array}{l}2,273,134- \\
2,338,024\end{array}$ & $61.2 \%$ \\
\hline Drug costs & 50,284 & $\begin{array}{l}2,509,235 \\
(1,757,068)\end{array}$ & $\begin{array}{l}2,493,877- \\
2,524,593\end{array}$ & $76.1 \%$ & 27,906 & $\begin{array}{l}2,659,022 \\
(1,812,180)\end{array}$ & $\begin{array}{l}2,637,760- \\
2,680,285\end{array}$ & $70.6 \%$ \\
\hline $\begin{array}{l}\text { Defined MTT } \\
\text { costs }\end{array}$ & 50,284 & $\begin{array}{l}1,895,454 \\
(1,617,171)\end{array}$ & $\begin{array}{l}1,881,319- \\
1,909,589\end{array}$ & $57.5 \%$ & 27,906 & $\begin{array}{l}1,912,357 \\
(1,665,631)\end{array}$ & $\begin{array}{l}1,892,814- \\
1,931,900\end{array}$ & $50.8 \%$ \\
\hline $\begin{array}{l}\text { Moisturizer } \\
\text { costs }\end{array}$ & 0 & - & - & & 20,656 & $4008(7,453)$ & $3906-4109$ & $0.1 \%$ \\
\hline $\begin{array}{l}\text { Other drug } \\
\text { costs }\end{array}$ & 49,564 & $\begin{array}{l}622,697 \\
(777,848)\end{array}$ & $\begin{array}{l}615,849- \\
629,545\end{array}$ & $18.9 \%$ & 27,864 & $\begin{array}{l}744,820 \\
(883,418)\end{array}$ & $\begin{array}{l}734,447- \\
755,193\end{array}$ & $19.8 \%$ \\
\hline $\begin{array}{l}\text { Radiotherapy } \\
\text { costs }\end{array}$ & 2893 & $\begin{array}{l}269,379 \\
(144,226)\end{array}$ & $\begin{array}{l}264,121- \\
274,637\end{array}$ & $8.2 \%$ & 2176 & $\begin{array}{l}274,839 \\
(161,086)\end{array}$ & $\begin{array}{l}268,067- \\
281,611\end{array}$ & $7.3 \%$ \\
\hline \multicolumn{9}{|c|}{ Entire 12 months of follow-up } \\
\hline $\begin{array}{l}\text { Total medical } \\
\text { costs }\end{array}$ & 50,284 & $\begin{array}{l}5,135,243 \\
(3,395,783)\end{array}$ & $\begin{array}{l}5,105,562- \\
5,164,925\end{array}$ & $100.0 \%$ & 27,906 & $\begin{array}{l}6,164,555 \\
(3,687,885)\end{array}$ & $\begin{array}{l}6,121,284- \\
6,207,826\end{array}$ & $100.0 \%$ \\
\hline $\begin{array}{l}\text { Outpatient } \\
\text { costs }\end{array}$ & 50,175 & $\begin{array}{l}3,535,744 \\
(3,039,421)\end{array}$ & $\begin{array}{l}3,509,149- \\
3,562,339\end{array}$ & $68.9 \%$ & 27,774 & $\begin{array}{l}3,593,280 \\
(2,962,900)\end{array}$ & $\begin{array}{l}3,558,433- \\
3,628,127\end{array}$ & $58.3 \%$ \\
\hline Inpatient costs & 34,939 & $\begin{array}{l}2,313,020 \\
(2,556,791)\end{array}$ & $\begin{array}{l}2,286,210- \\
2,339,831\end{array}$ & $45.0 \%$ & 22,995 & $\begin{array}{l}3,141,044 \\
(3,418,671)\end{array}$ & $\begin{array}{l}3,096,856- \\
3,185,233\end{array}$ & $51.0 \%$ \\
\hline \multicolumn{9}{|l|}{ Drug costs } \\
\hline $\begin{array}{l}\text { Defined MTT } \\
\text { costs }\end{array}$ & 50,284 & $\begin{array}{l}2,960,789 \\
(2,772,164)\end{array}$ & $\begin{array}{l}2,936,559- \\
2,985,020\end{array}$ & $57.7 \%$ & 27,906 & $\begin{array}{l}3,079,287 \\
(2,722,818)\end{array}$ & $\begin{array}{l}3,047,340- \\
3,111,235\end{array}$ & $50.0 \%$ \\
\hline $\begin{array}{l}\text { Moisturizer } \\
\text { costs }\end{array}$ & 0 & - & - & & 27,906 & $6033(12,344)$ & $5888-6178$ & $0.1 \%$ \\
\hline $\begin{array}{l}\text { Other drug } \\
\text { costs }\end{array}$ & 49,808 & $\begin{array}{l}979,841 \\
(1,291,595)\end{array}$ & $\begin{array}{l}968,498- \\
991,184\end{array}$ & $19.1 \%$ & 27,898 & $\begin{array}{l}1,246,769 \\
(1,469,595)\end{array}$ & $\begin{array}{l}1,229,524- \\
1,264,015\end{array}$ & $20.2 \%$ \\
\hline $\begin{array}{l}\text { Radiotherapy } \\
\text { costs }\end{array}$ & 4284 & $\begin{array}{l}283,912 \\
(151,318)\end{array}$ & $\begin{array}{l}279,379- \\
288,444\end{array}$ & $5.5 \%$ & 3517 & $\begin{array}{l}289,301 \\
(166,077)\end{array}$ & $\begin{array}{l}283,811- \\
294,792\end{array}$ & $4.7 \%$ \\
\hline \multicolumn{9}{|c|}{$\begin{array}{l}\text { Note: Of } 20,709 \text { patients in the study cohort, } 12 \text { patients }(0.043 \%) \text { had no cost data for moisturizers; therefore, these patients were excluded } \\
\text { from the analysis. }\end{array}$} \\
\hline
\end{tabular}

We observed an increasing trend in both the proportion of moisturizer users and the amount of moisturizer used per patient over time (Online Resource 6).

Overall, changes in the proportion of moisturizer users over time in the MTT groups with $\geq 1000$ patients showed a continuous year-to-year increase (Online Resource 7).

Discussion 
As of 2019 , the MDV database covered $22 \%$ of acute-phase medical institutions in the DPC system in Japan. Provided that all cancer patients are seen at acute-phase medical institutions, we estimate that approximately 870,000 cancer patients were treated with MTT during the follow-up period in this study (the number of patients included as the source cohort [190,536] divided by 0.22 ). The Ministry of Health, Labour, and Welfare in Japan estimated that as of 2017 , there were $1,782,000$ cancer patients. Considering that most anti-cancer drugs developed since 2000 are MTTs, "patients treated with MTT" who were included in our study cohort are expected to account for a large proportion of cancer patients.

\section{Moisturizer utilization pattern}

The proportion of patients prescribed moisturizers was 35.7\% among patients prescribed MTT during the study period. The proportion varied greatly among the MTT groups. The proportion of moisturizer users was the highest in the EGFR inhibitor group (71.4\%) followed by the multikinase inhibitor group (50.2\%), immune check point inhibitor group (42.1\%), and the VEGF inhibitor group (40.2\%). The proportion of users was low in the anti-CD20 antibody group (23.2\%) and the BCR-ABL inhibitor group (15.4\%). The proportion of moisturizer users over time showed an increasing trend (31.6\% in 2011 to $39.1 \%$ in 2018), and the proportion of moisturizer users in the EGFR inhibitor group increased from 53.5$74.7 \%$ during the same period. Moreover, in the 2018 data, the proportion of moisturizer users in the BCR-ABL inhibitor group, which was the lowest among the MTT groups, reached $21.1 \%$. These findings suggest that moisturizer use has become common not only when EGFR inhibitors are used, which commonly cause skin disorders because of their mechanisms of action and for other reasons, but also when other anti-cancer drugs are used. The proportion of EGFR inhibitors among the MTT groups actually decreased (13.8\% in 2012 to 5.8\% in 2018), which suggests that the increasing trend in moisturizer use was not caused by changes in the type of MTT being prescribed.

\section{Variations in the moisturizer utilization pattern}

In Group M, 31.5\% of the patients were prescribed moisturizers within 1 month after beginning MTT. However, the start of prescriptions was not limited to a certain period. Frequent symptoms of skin disorder differ depending on the MTT. Symptoms such as acneiform rash peaked approximately 2 weeks after beginning MTT, while other symptoms, such as xerosis and paronychia, mainly developed after 28 days or later [10, 11]. These findings suggest that variable timing of initiating moisturizer use in the present analysis reflects variable timing in the onset of skin disorders.

\section{Overall budget impact of moisturizers}

When drugs were compared according to their unit prices, there was a large difference between moisturizers and MTTs. Therefore, when we evaluated medical costs and drug costs per patient, the contribution of moisturizer costs was small. Specifically, the analysis of total medical costs at 6 months and 12 months showed that the difference in medical costs between Group M and Group N was 0.467 million yen at 6 months and 1.029 million yen at 12 months. However, the difference in moisturizer costs was 4008 yen at 6 months and 6033 yen at 12 months, which accounted for $0.1 \%$ of the total medical costs and only $0.6 \%$ in the difference in the total costs at 12 months.

The total medical costs for users of anti-cancer drugs in Japan, calculated by dividing the total medical costs of users of anti-cancer drugs in the MDV database by $22 \%$, which is the patient coverage of this database, was 1.96 trillion yen (1.17 trillion yen for non-users of moisturizers and 0.87 trillion yen for users); moisturizer costs accounted for 760 million yen. Therefore, conceivably, the medical costs of prescribed moisturizers in cancer treatment is extremely low. The main factors causing the high medical costs in Group M were extended hospitalization and increased costs of drugs other than moisturizers.

\section{Limitations}

The present study used commercially available claims data from DPC hospitals and did not capture treatments performed in non-DPC hospitals or clinics. Follow-up of patients who were transferred to other medical institutions during treatment was also not possible. In Japan, unlike in other countries, the availability of a health insurance claims database at the national level is extremely limited. However, some drug utilization studies using commercially available claims data have been reported despite the limitations $[12,13,14]$. We expect that similar analyses will be performed in the future using a broader database, to improve the generalizability of the findings in this study, if such a database becomes available.

The present study was not a comprehensive analysis of cancer treatment. We analyzed only cancer patients who were prescribed MTT. Some MTTs, for example, EGFR inhibitors, are expected to cause skin disorders frequently because of their mechanisms of action. Therefore, the need for moisturizers in patients receiving MTT are higher than for patients receiving other anti-cancer drugs, which was why patients receiving MTT were chosen as the first cohort. However, because cytotoxic anti-cancer drugs can also cause skin disorders as an adverse event, broader studies analyzing patients receiving a wide variety of anti-cancer drugs is desirable.

When we selected the patients for this study cohort from the source cohort, we excluded patients who were prescribed moisturizers before initiating MTT. This criterion was set to exclude the use of moisturizers to treat skin disorders not caused by anti-cancer drugs. However, this criterion also excluded patients prescribed moisturizers prophylactically and those who were already being treated with other anti-cancer drugs

Page 9/12 
and who were prescribed moisturizers for skin disorders. Because of the stricter inclusion/exclusion criteria we set to identify only patients strongly associated with skin disorders caused by MTT, we expect that the number of patients using moisturizers in clinical practice is higher.

\section{Conclusion}

In this study, we analyzed moisturizer use in patients treated with MTT, using a commercially available claims database. The proportion of patients treated with MTT who used moisturizers was 35.7\%, and the proportion varied among the MTT groups. The proportion of patients who were prescribed moisturizers increased over time. We also saw variations in the prescription length in days and the amount of moisturizers prescribed. Thus, we believe that moisturizers were used according to each patient's condition.

Regarding the medical costs required for MTT treatment, the medical costs in Group M were higher than for Group N by 1.029 million yen. However, most of the costs resulted from costs not related to the moisturizer costs (e.g., costs for hospitalization and other drugs). The difference in costs that can be associated with moisturizers was approximately 6000 yen. Moisturizers accounted for $0.6 \%$ of the difference in the total medical costs in Group $\mathrm{N}$ and $0.1 \%$ of the total medical costs. We determined that the cost and use of moisturizers may contribute to continuation of MTT treatment.

\section{Declarations}

\section{Funding}

This study was funded by Maruho Co., Ltd.

\section{Conflict of interest/competing interests}

This study was funded by Maruho Co., Ltd. Creativ-Ceutical K.K., Tokyo, Japan was paid to conduct the study. Ataru Igarashi and Yoshio Kiyohara received lecture/consultation fees from Maruho Co., Ltd.; Anastasiia Motrunich and Yoshie Onishi are employee of Creativ-Ceutical.; Akira Kanakubo and Momoyo Kishida are employees of Maruho Co., Ltd.; Lida Teng and Toshiya Matsuzaki have no conflict of interest.

\section{Ethics approval}

Not applicable

\section{Consent to participate}

Not applicable

\section{Consent to publish}

Not applicable

\section{Availability of data and materials}

Not available because the datasets are commercial data of Medical Data Vision, Co., Ltd.

\section{Authors' contributions}

All authors contributed to the study conception and design. Statistical analyses were performed by Yoshie Onishi and Anastasiia Motrunich. The first draft of the manuscript in Japanese was written by Ataru Igarashi and Yoshio Kiyohara. All authors read and approved the final manuscript.

\section{Acknowledgment}

Language editing assistance was provided by ASCA corporation

\section{References}

1. Lacouture ME, Anadkat MJ, Bensadoun RJ, Bryce J, Chan A, Epstein JB, Eaby-Sandy B, Murphy BA, MASCC Skin Toxicity Study Group (2011) Clinical practice guidelines for the prevention and treatment of EGFR inhibitor-associated dermatologic toxicities. Support Care Cancer 19:1079-1095. https://doi.org/10.1007/s00520-011-1197-6

2. National Cancer Center Japan, ed (2016) 2016 Clinical Guide of Appearance Care for People Receiving Cancer Treatment [in Japanese]. Tokyo, Kanehara \& Co., Ltd. 
3. Grande R, Narducci F, Bianchetti S, Mansueto G, Gemma D, Sperduti I, Trombetta G, Angelini F, Gamucci T (2013) Pre-emptive skin toxicity treatment for anti-EGFR drugs: evaluation of efficacy of skin moisturizers and lymecycline. A phase II study. Support Care Cancer 21:16911695. https://doi.org/10.1007/s00520-012-1715-1

4. Watanabe S, Nakamura M, Takahashi H, Hara M, ljichi K, Kawakita D, Morita A (2017) Dermopathy associated with cetuximab and panitumumab: investigation of the usefulness of moisturizers in its management. Clin Cosmet Investig Dermatol 11:353361. https://doi.org/10.2147/ccid.s140796

5. Sekiguchi K, Ogita M, Akahane K, Haga C, Ito R, Arai S, Ishida Y, Tsukada Y, Kawamori J (2015) Randomized, prospective assessment of moisturizer efficacy for the treatment of radiation dermatitis following radiotherapy after breast-conserving surgery. Jpn $\mathrm{J}$ Clin Oncol. 45:1146-1153. https://doi.org/10.1093/jjco/hyv155

6. Sekiguchi K, Akahane K, Ogita M, Haga C, Ito R, Arai S, Ishida Y, Tsukada Y, Kawamori J (2018) Efficacy of heparinoid moisturizer as a prophylactic agent for radiation dermatitis following radiotherapy after breast-conserving surgery: a randomized controlled trial. Jpn $\mathrm{J}$ Clin Oncol. 48:450-457. https://doi.org/10.1093/jjco/hyy045

7. Yoshida Y, Hashimoto K, Saeki H, Itagaki F, Hirama A, Suzuki A, liyama K, Fukunaga Y, Enomoto H, Kushima E, Kishida M, Fujimoto S, Tsuruoka S (2019) Efficacy of a Moisturizer for Pruritus Accompanied With Asteatosis in Dialysis Patients: An Open-Label, Randomized, Exploratory Study. Kidney Med. 1:191-199. https://doi.org/10.1016/j.xkme.2019.04.008

8. Wacker B, Nagrani T, Weinberg J, Witt K, Clark G, Cagnoni PJ (2007) Correlation between development of rash and efficacy in patients treated with epidermal growth factor receptor tyrosine kinase inhibitor erlotinib in two large phase III studies. Clin Cancer Res 13:39133921. https://doi.org/10.1158/1078-0432.ccr-06-2610

9. Sugiura Y, Nemoto E, Kawai O, Ohkubo Y, Fusegawa H, Kaseda S (2013) Skin rash by gefitinib is a sign of favorable outcomes for patients of advanced lung adenocarcinoma in Japanese patients. Springerplus 2:22. https://doi.org/10.1186/2193-1801-2-22

10. Beech J, Germetaki T, Judge M, Paton N, Collins J, Garbutt A, Braun M, Fenwick J, Saunders MP (2018) Management and grading of EGFR inhibitor-induced cutaneous toxicity. Future Oncol 14: 2531-2541. https://doi.org/10.2217/fon-2018-0187

11. Kozuki T (2016) Skin problems and EGFR-tyrosine kinase inhibitor. Jpn J Clin Oncol 46: 291-298. https://doi.org/10.1093/jjco/hyv207

12. Sruamsiri R, Iwasaki K, Tang W, Mahlich J (2018) Persistence rates and medical costs of biological therapies for psoriasis treatment in Japan: a real-world data study using a claims database. BMC Dermatol 18:5. https://doi.org/10.1186/s12895-018-0074-0

13. Igarashi A, Fujita H, Arima K, Inoue T, Dorey J, Fukushima A, Taguchi Y (2019) Health-care resource use and current treatment of adult atopic dermatitis patients in Japan: A retrospective claims database analysis. J Dermatol 46:652-661. https://doi.org/10.1111/1346-8138.14947

14. Imafuku S, Matsuki T, Mizukami A, Goto Y, de Souza S, Jégou C, Bianco V, Rosillon D, Ito C, Curran D, Holl K (2019) Burden of herpes zoster in the Japanese population with immunocompromised/chronic disease conditions: results from a cohort study claims database from 20052014. Dermatol Ther (Heidelb) 9:117-133. https://doi.org/10.1007/s13555-018-0268-8

\section{Figures}




\section{Total excluded patients: $n=112,346^{\dagger}$}

- Not treated with the defined MTT during the selection period, $n=41,134$

- Prescribed at least one moisturizer (heparinoid, vaseline, urea) within 6 month sefore the index date, $n=$ 27,653

- Treated with the defined MTT within 6 months before the index date, $n=$ 3236

- Not continuously observed in database during the follow-up period (patients must have at least 1 medical claim each quarter within the followup period), $n=46,777$

- Aged $<18$ years old at the index date, $n=358$

- Prescribed MTT drugs from more than one MTT category at the index date, $n=177$

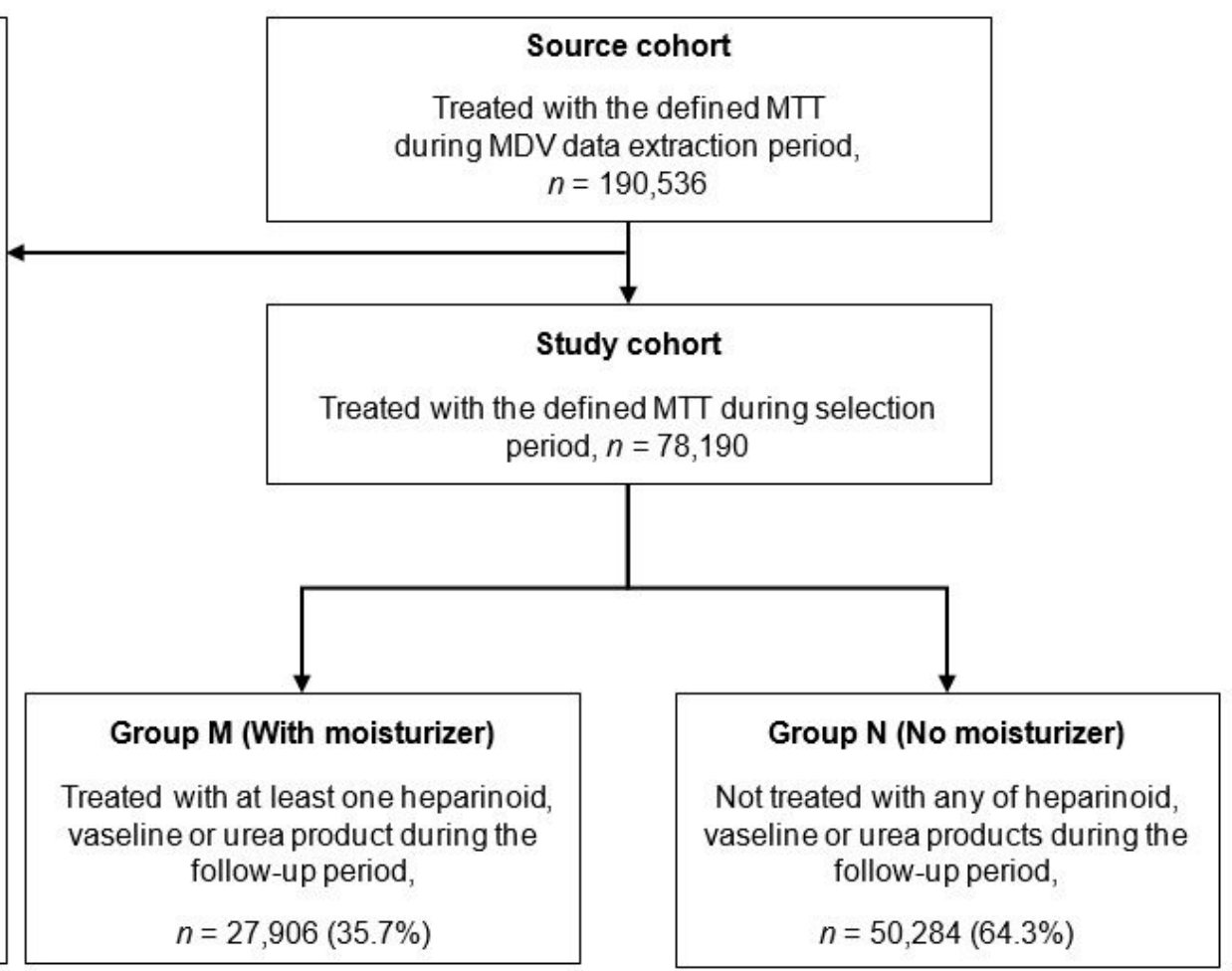

\section{Figure 1}

Study flowchart. †Patients were excluded for several reasons; therefore, the sum of the excluded patients is greater than the total number of excluded patients. MTT molecularly targeted therapy Data extraction period: April 2008 to April 2019 Index date: Date of the first MTT prescription Selection period: October 2011 to April 2018 Follow-up period: 1 year from the index date

\section{Supplementary Files}

This is a list of supplementary files associated with this preprint. Click to download.

- ESM1.docx 